\title{
FRAGILIDADES DO SERVIÇO PÚBLICO ODONTOLÓGICO NA ÓTICA DE CIRURGIÕES-DENTISTAS DO MUNICÍPIO DE PONTA GROSSA, PARANÁ, BRASIL
}

\section{WEAKNESSES IN PUBLIC DENTAL SERVICE IN A DENTIST PERSPECTIVE OF PONTA GROSSA MUNICIPALITY, PARANA, BRAZIL}

\author{
Danielle Bordin ${ }^{1 *}$, Cristina Berger Fadel ${ }^{2}$, Cristiane G. Souza ${ }^{3}$, Cléa Adas Saliba \\ Garbin $^{1}$, Suzely Adas Saliba Moimaz ${ }^{1}$, Nemre Adas Saliba ${ }^{1}$ \\ ${ }^{1}$ Universidade Estadual Paulista, Departamento de Odontologia Preventiva e Social, \\ Araçatuba, São Paulo, Brasil. ${ }^{2}$ Universidade Estadual de Ponta Grossa, Departamento \\ de Odontologia, Ponta Grossa, Paraná, Brasil. ${ }^{3}$ Universidade Estadual de Ponta Grossa, \\ Programa de Pós-graduação em Ciências Sociais Aplicadas, Ponta Grossa, Paraná, Brasil.
}

*Autor correspondente: Departamento de Odontologia Preventiva e Social. FOA/UNESP. Rua José Bonifácio, 1193 - Seção de Pós-Graduação, CEP: 16015-050, Vila Mendonça, Araçatuba - SP. Brasil. E-mail: daniellebordin@hotmail.com

\section{RESUMO}

Este estudo tem por objetivo avaliar a percepção de cirurgiões-dentistas sobre as necessidades de melhorias no serviço público odontológico. Para a sua realização, participaram 30 cirurgiões-dentistas pertencentes ao serviço público odontológico da Atenção Primária em Saúde do município de Ponta Grossa, Paraná, Brasil. Os entrevistados foram investigados individualmente por meio de formulário com questões abertas. As respostas foram transcritas integralmente e submetidas à Técnica da Análise de Conteúdo resultando em seis categorias. A categoria estrutura física e equipamentos foi a que recebeu o maior número de implicações, sendo o espaço reduzido do consultório e a falta de equipamento de raios- $X$ as principais críticas. A segunda categoria foi a necessidade de ampliação de recursos humanos, seguida de maior investimento em ações de cunho preventivo e capacitação da equipe. Estas percepções devem ser consideradas pelos gestores locais com vistas a realizar um planejamento estratégico que possa contemplar adequadamente as lacunas expostas, propiciando um serviço odontológico de qualidade.

Palavras-chave: Avaliação de Serviços de Saúde. Atenção Primária à Saúde. Saúde Bucal. Pessoal de Saúde.

\begin{abstract}
The aim of this study was to evaluate the perception of dentists on the need for improvements in the public dental service. For its implementation, participated 30 dentists belonging to public dental services of primary health care in the city of Ponta Grossa, Paraná, Brazil. Respondents were investigated individually through open questions form. The answers were transcribed and submitted to the Technique of Content Analysis. The analysis resulted in six categories. The physical structure and equipment category was one that showed the largest number of suggestions, and the small space of the clinic and lack of X-ray equipment the main criticisms. The second was the need to expand human resources, followed by greater investment in preventive actions and staff training. These suggestions should be reviewed by local managers in order to carry out a strategic plan that can adequately address these gaps and provide a dental service quality.

Keywords: Evaluation of Health Services. Primary Health Care. Oral Health. Health Personnel.
\end{abstract}




\section{INTRODUÇÃO}

O serviço de atenção à saúde bucal dentro do Sistema Único de Saúde, apesar de ter angariado grandes avanços nos últimos anos, através da implementação de uma política própria (BRASIL, 2004) com a ampliação e inserção de equipes na Estratégia Saúde da Família e fortalecimento de suas redes de atenção (BRASIL, 2004; 2012), continua enfrentando diversos obstáculos para a efetivação de suas ações com qualidade, especialmente no que tange aos recursos físicos, humanos e tecnológicos (SOARES, 2007). Neste sentido, continua sendo considerado um componente crítico do sistema de saúde (VAN STRALEN et al., 2008), e poucas estratégias são articuladas para minimizar este desafio.

Deste modo, como meio de fomentar o enfrentamento deste desafio, é necessário compreender a fundo e da forma mais concreta possível, quais são os aspectos que inferem negativamente na conquista de um serviço odontológico de qualidade. Configura-se como uma das estratégias de apreensão destes fatores a análise dos profissionais envolvidos neste processo, os cirurgiões-dentistas, que vivenciam rotineiramente as realidades intrínsecas a esse contexto.

A compreensão destes atores é exposta como fator de provimento de informações sobre os problemas cotidianos dos serviços, propiciando avanços no âmbito da produção de cuidados e gestão de ações (NORA, JUNGES, 2013; BRANDÃO, et al., 2013; MOHAMMED, et al., 2014; CONSTAND, et al. 2014; GOETZ, et al., 2014; BORDIN et al., 2016), favorecendo a tomada assertiva de decisões e induzindo melhoria na oferta de serviços em termos de qualidade.

Assim sendo, é notória a importância do desenvolvimento de pesquisas voltadas para o entendimento de cirurgiões-dentistas acerca das fragilidades do serviço público odontológico, como um abastado meio de fomentar avanços na organização e planejamento das estratégias em saúde bucal (BORDIN, 2014).

Contudo, os estudos desenvolvidos na área da Odontologia expõem, em sua maioria, uma abordagem reducionista, restrita ao aspecto da contenção de custos (GOETZ, et al., 2014), em detrimento à qualidade dos serviços. Fica evidente a necessidade urgente de adotar ferramentas metodológicas e operacionais efetivas em serviços e programas de saúde bucal, aptas a subsidiar processos de decisão e que possam servir de suporte para o redirecionamento das práticas de saúde.

Por estas considerações e pela ciência de que o objeto exposto deve estar cada vez mais presente na agenda do gestor em saúde, este estudo objetiva avaliar a percepção de cirurgiões-dentistas sobre as necessidades de melhorias no serviço público odontológico.

\section{METODOLOGIA}

\section{Descrição do estudo}

Trata-se de um estudo de caráter transversal, do tipo inquérito, com a abordagem qualitativa, realizado no município de Ponta Grossa, no estado do Paraná. A pesquisa foi aprovada pelo Comitê de Ética em Pesquisas com Seres Humanos da Universidade Estadual de Ponta Grossa - UEPG (parecer $\mathrm{n}^{\circ}$ 799.292/2014), respeitando os ditames da resolução 466/12 do Conselho Nacional de Saúde Brasileiro.

\section{Local de estudo e amostra}

O município de Ponta Grossa contava, em 2014, com uma rede assistencial organizada, na Atenção Primária à Saúde (APS), com 40 unidades de saúde, sendo 17 Unidades Básicas de Saúde (UBS) e 23 Unidades de Saúde da Família (USF). Especificamente com relação à saúde bucal no âmbito do SUS, a construção da rede de serviços públicos é dada por meio de 43 pontos de atendimento odontológico: 17 situados em UBS, 01 clínica do bebê, 08 escolas/convênios, 03 unidades em zona rural e 14 equipes de saúde bucal na Estratégia Saúde da Família. Nestes 43 pontos existem 50 cirurgiões-dentistas, pois há locais com atendimento em dois turnos.

$\mathrm{O}$ estudo foi realizado entre os meses de setembro e outubro de 2014. No momento do estudo, a atenção odontológica do município em nível de APS era prestada por meio de 27 unidades regulares de saúde, subdivididas em unidades básicas de saúde e unidades de saúde da família.

A amostra foi composta pela totalidade de cirurgiões-dentistas $(\mathrm{n}=50)$ adscritos às unidades de saúde da Atenção Primária à Saúde do município investigado. 


\section{Coleta e análise dos dados}

Para coleta de dados realizou-se entrevista individual, na sala de reuniões ou local apropriado dos diferentes unidades de saúde. O entrevistador foi um cirurgião-dentista, devidamente calibrado a fim evitar vieses decorrentes de um possível interferência do entrevistador nas respostas do entrevistado, que respeitou os ditames éticos e legais para desenvolvimento de pesquisa com seres humanos.

Todos os entrevistados foram informados sobre o objetivo da pesquisa, seu caráter de voluntariedade e de não-identificação, assim como a forma de coleta, análise e destino dos dados. Os que assentiram com sua participação, o fizeram por meio da assinatura de um termo de consentimento livre e esclarecido.

Para o presente estudo foi investigado o perfil dos profissionais e a questão norteadora referente as necessidades de melhorias no atendimento e no ambiente odontológico da unidade de saúde investigada. As respostas foram transcritas integralmente e submetidas à técnica da análise de conteúdo, propostas por Bardin, (2004), com o intuito de qualificar as vivências dos cirurgiões-dentistas, bem como suas percepções sobre o serviço odontológico em investigação (BARDIN, 2004).

\section{Técnica de análise de conteúdo}

A técnica de análise de conteúdo é composta por procedimentos sistemáticos que proporcionam a descrição das mensagens, bem como as inferências sobre os dados coletados (BARDIN, 2004).

Inicialmente realizou-se a leitura flutuante do material obtido, para a apreensão integral do sentido posterior exploração, através de leituras sistemáticas, esta etapa é considerada por Bardin como pré-análise (BARDIN, 2004). Em seguida, realizou-se leitura exaustiva do material, separação entre os temas a serem trabalhados, e formularam-se pressupostos iniciais, em conformidade aos objetivos do estudo (BARDIN, 2004). Na etapa seguinte, descrita como exploração do material, foram extraídas seis categorias. Por fim, o material foi organizado de acordo com as categorias estabelecidas direcionando a análise propriamente dita (BARDIN, 2004).

Os resultados foram sintetizados em um quadro, apontando: a categoria, o número de entrevistados e unidade de contexto, que descreve excertos representativos da categoria em questão.

\section{RESULTADOS}

A amostra final foi composta 30 cirurgiões-dentistas, sendo 19 do gênero feminino e 11 do gênero masculino, com faixa etária média de 48 anos. As perdas dos sujeitos ocorreram pela ausência do profissional nos dias de entrevista, por motivo de férias ou de licença médica.

O quadro 01 apresenta a percepção dos cirurgiões-dentistas sobre aspectos que precisam ser melhorados no serviço público odontológico no âmbito da Atenção Primária à Saúde. 
Quadro 01. Percepção de cirurgiões-dentistas acerca das necessidades de melhorias no serviço público odontológico (n=30). Ponta Grossa, Paraná, 2014

\begin{tabular}{|c|c|c|}
\hline Categoria & $\mathbf{N}^{1}$ & Unidade de contexto \\
\hline $\begin{array}{l}\text { Estrutura física e } \\
\text { equipamentos }\end{array}$ & 12 & $\begin{array}{l}\text { "O consultório é muito pequeno para o atendimento". } \\
\text { "Melhora nas condições de trabalho: no verão o calor é insuportável, no inverno o frio é } \\
\text { insuportável". } \\
\text { "No atendimento seria ideal um aparelho de Raio-X". } \\
\text { "Precisa de mesas auxiliares, balcão ou armários. A mobilia é muito velha e improvisada". }\end{array}$ \\
\hline $\begin{array}{l}\text { Ampliação de } \\
\text { Recursos humanos }\end{array}$ & 11 & $\begin{array}{l}\text { "Como são duas equipes de saúde da família e só uma equipe de saúde bucal, são muitos } \\
\text { usuários e apenas um dentista, ou seja, deveria ser uma equipe para um dentista". } \\
\text { "Ter dentista em horário noturno para atender os pacientes que trabalham". } \\
\text { "Ter dentista no periodo da tarde, para o atendimento de crianças e adolescentes que } \\
\text { estudam pela manhã, que faltam nas aulas, ou então, não comparecem quando não podem } \\
\text { faltar aula neste dia agendado". } \\
\text { "Que a equipe pudesse trabalhar a quatro mãos dinamizando o atendimento, em ambiente } \\
\text { saudável, alegre". }\end{array}$ \\
\hline $\begin{array}{l}\text { Ampliação de ações } \\
\text { de cunho preventivo }\end{array}$ & 09 & $\begin{array}{l}\text { "Ter horário para realizar a promoção da saúde". } \\
\text { "Investir mais na prevenção para a população e para os escolares, dando a eles a } \\
\text { possibilidade de usar a prevenção contra os males que podem ocorrer na sua saúde bucal". } \\
\text { "Oferecer durante o atendimento, principalmente para crianças, folders informativos com } \\
\text { desenhos para colorir enfatizando a saúde bucal e entregar escovas. Antes isso era possivel } \\
\text { e as crianças adoravam, agora nos pedidos de material é uma raridade!" }\end{array}$ \\
\hline $\begin{array}{l}\text { Capacitação de } \\
\text { recursos humanos }\end{array}$ & 04 & $\begin{array}{l}\text { "Tempo para realizar atualizações no campo da saúde bucal". } \\
\text { "Melhorar estratégias para termos equipes coesas, com a mesma intenção, voltada para } \\
\text { a mesma finalidade de proporcionar boas condiçôes de saúde em ambiente acolhedor". }\end{array}$ \\
\hline Cultura do Paciente & 02 & $\begin{array}{l}\text { "Pacientes deveriam avisar quando não pudessem comparecer à consulta para que a vaga } \\
\text { seja destinada à outra pessoa". }\end{array}$ \\
\hline $\begin{array}{l}\text { Não necessita de } \\
\text { melhorias }\end{array}$ & 02 & $\begin{array}{l}\text { "O ambiente é apropriado para o atendimento com equipamento em bom funcionamento e } \\
\text { no momento está atendendo a necessidade da população". }\end{array}$ \\
\hline
\end{tabular}

${ }^{1}$ Refere-se ao número de entrevistados que fez inferência a categoria em questão.

\section{DISCUSSÃO}

Ao analisar os excertos dos cirurgiões-dentistas no que tange a necessidade de melhorias no serviço público odontológico no âmbito da APS verificou-se que a categoria estrutura física e equipamentos foi a que apontou o maior número de sugestões, sendo as principais críticas direcionadas ao espaço reduzido do consultório odontológico e à falta de equipamento de raios-X odontológico.

Os achados aqui expostos corroboram com o estudo de Nora e Junges em 2013, o qual verificou que a precariedade das instalações nas unidades de saúde apareceu como um conteúdo recorrente em diferentes estudos, sendo os principais problemas apontados as áreas físicas muito pequenas, a pouca ventilação e os problemas na conservação da estrutura física.
Estudos mostram que problemas nestes aspectos estruturais interferem diretamente no processo de trabalho, comprometem a plena utilização da capacidade instalada dos serviços e bem como sua qualidade (PEDROSA, et al. 2011; TAVARES, et al. 2013; NORA, JUNGES 2013). Ainda, geram desmotivação em profissionais e gestores, além de desconforto a todos os atores que rotineiramente trabalha e usufruem do serviço (NORA, JUNGES 2013).

Dispor de uma estrutura física apropriada, com disponibilidade de equipamentos adequados e de materiais e insumos suficientes à assistência prestada, propicia ao serviço de saúde a promoção de local digno, capaz de fomentar a atenção de modo mais acolhedor e humano, tanto para trabalhadores quanto para usuários, favorecendo assim maior qualidade de assistência (BRASIL, 2008; RICCI et al., 2011; BRASIL, 2012). 
A grande preocupação exposta pelos cirurgiões-dentistas entrevistados com os fatores que afetam diretamente o desenvolvimento de suas funções destaca a necessidade urgente de ampliação e implementação de novas unidades de saúde, com vistas a subsidiar condições mais adequadas às necessidades de profissionais e pacientes.

Outra categoria que recebeu destaque refere-se à carência de recursos humanos, demonstrando que a cobertura assistencial no nível primário ainda é baixa, sendo congruente com diversos estudos (MOIMAZ, et al., 2010; GONÇALVES, 2011; BRANDÃO et al., 2013), mesmo com a expansão expressiva da Estratégia Saúde da Família em todo o país.

A principal sugestão dos entrevistados foi igualar o número de equipes de saúde bucal ao número de equipes de saúde médica nos diferentes locais de atendimento. Com esta estratégia seria possível ter uma clientela adscrita adequada, e diminuir a demanda reprimida em saúde bucal existente no município investigado.

Os achados convergem com a literatura ao passo que a disponibilidade e a qualidade dos recursos humanos, a oferta de serviços e sua distribuição geográfica, afetam de maneira significativa o acesso (BRANDÃO, et al., 2013), levando a grandes demandas de atendimento, causando sofrimento aos profissionais e usuários (NORA, JUNGES, 2013).

Os entrevistados sugerem ainda, a necessidade de implementação de equipes de saúde bucal em período noturno para atender a demanda de usuários trabalhadores. O apontamento é reflexo da grande dificuldade encontrada por usuários que trabalham em tempo integral, tendo que no horário de funcionamento regular das unidades de saúde afastar-se de suas atividades laborais para cuidar da saúde bucal (KONTOPANTELIS et al., 2010).

Deste modo, gestores e profissionais da saúde devem implantar uma agenda planejada de ações e serviços em saúde, com ênfase na oferta de períodos alternativos de atendimento e de agendamento de consultas (MOIMAZ et al., 2015), considerando também os períodos noturnos e os finais de semanas.

Outras necessidades de melhoria elencadas pelos cirurgiões-dentistas refere-se à ampliação da disponibilidade para planejamento e realização de ações preventivo-educativas de cunho coletivo e individual, e a maiores investimentos para aquisição de produtos que visam à prevenção de agravos em saúde bucal.

A supervalorização da assistência curativa em detrimento a atenção preventiva, ainda está fortemente arraigada no pensamento de muitos gestores, profissionais e pacientes, condição que dificulta o planejamento e desenvolvimento adequado de ações promotoras de saúde. Esta lógica precisa ser alterada e também ser ofertado mais espaço a estratégias que tragam, de fato, mudanças no estilo de vida das pessoas assistidas pelos serviços de saúde, potencializando sua qualidade de vida.

Para tanto, é necessário que todos os atores evolvidos no processo de cuidado em saúde, mobilizem-se para atuar em conjunto, utilizando-se dos mais diversos recursos, com motivações constantes, de caráter permanente, promovendo a aprendizagem consciente e agradável, pois o simples contato com informações nem sempre é suficiente para garantir um comportamento satisfatório e duradouro sobre os hábitos saudáveis de saúde bucal (ALVES et al., 2014).

Outro apontamento apreendido diz respeito à importância de intensificar estratégias de capacitação profissional com vistas à atualização científica e realização de atendimento de forma integral humanizada. Estas estratégias são importantes para uma conduta profissional comprometida, participativa, humana e com melhor capacidade técnica, para a edificação e a consolidação de um atendimento com melhor qualidade e congruente às necessidades do paciente (NORA, JUNGES, 2013; MOIMAZ et al., 2016).

De forma pouco frequente, entrevistados apontaram a necessidade de mudança de conduta dos usuários do serviço odontológico em relação à desmarcação de consulta na impossibilidade de comparecimento, podendo desta forma, destinar o horário de atendimento a outra pessoa. Esta sugestão compõe uma estratégia capaz de otimizar os serviços, diminuindo as faltas, o tempo ocioso do profissional e a demanda reprimida. Para que esta conduta seja de fato corriqueira nos serviços de saúde, é necessária a atuação dos diferentes profissionais da saúde, para expor ao usuário a importância de não faltar ou avisar com antecedência o não comparecimento. Os usuários podem ser informados através de cartazes, exposições dialogadas em reuniões de grupo e durante o processo de agendamento.

Apesar das sugestões levantadas, alguns indivíduos revelaram não haver necessidade de 
aprimoramentos nos serviços públicos odontológicos investigados. Este resultado coadunam com estudos de Gonçalves (2011) e Bordin (2016), o qual pontua que os entrevistados consideram as condições físicas e estruturais das unidades de saúde que frequentam satisfatórias e adequadas.

\section{Limitações do estudo}

Por se tratar de uma amostra por conveniência e de apenas um município brasileiro, esta não pode ser representativa de todas as práticas de cuidados em saúde bucal do Brasil. No entanto, a literatura aponta dados bastante semelhantes em diferentes regiões brasileiras. Ainda, por ser um estudo exploratório não é possível extrair conclusões sobre causalidade e por esta razão os resultados devem ser interpretados com ressalvas.

\section{CONCLUSÃO}

Frente ao exposto, conclui-se que as principais necessidades de melhorias nos serviços odontológicos investigados referem-se à ampliação da estrutura física e disponibilidade de equipamentos, ampliação e capacitação de recursos humanos e necessidade de maior investimento em ações de cunho preventivo. As sugestões dos cirurgiões-dentistas devem ser consideradas pelos gestores locais com vistas a realizar um planejamento estratégico que possa contemplar adequadamente as lacunas expostas e propiciar um serviço odontológico de qualidade.

\section{REFERÊNCIAS}

ALVES, F.B.T; et al. Infant motivation in dental health: Attitude without constant reinforcement. Journal of Indian Society of Pedodontics and Preventive Dentistry. v.32, n. 3, p. 225-230. 2014.

BARDIN L. Análise de conteúdo. $3^{\text {a }}$ ed. Lisboa: Edições 70, 2004.

BORDIN, D. Qualidade do Serviço Público Odontológico no Brasil: a percepção de usuários e profissionais da saúde. Dissertação de Mestrado. Araçatuba, 2014. 88 f.

BORDIN, D. et al. Estudo comparativo da satisfação de usuários e profissionais da saúde com o serviço público odontológico. Cienc Saude Colet. Artigo no prelo. Disponível em: http://www.cienciaesaudecoletiva.com.br/ artigos/artigo_int.php?id_artigo=15343 Acesso em: 20 jul 2016.
BRANDÃO, A.L.R.B.S.; GIOVANELLA, L; CAMPOS, C.E.A. Avaliação da atenção básica pela perspectiva dos usuários: adaptação do instrumento EUROPEP para grandes centros urbanos brasileiros. Cienc Saude Colet., v.18, n.1, p.103-114, 2013.

BRASIL. Ministério da Saúde. Secretaria de Atenção à Saúde. Departamento de Atenção Básica. Manual de estrutura física das unidades básicas de saúde: saúde da família. Secretaria de Atenção à Saúde. 2. ed. Brasília (DF): Ministério da Saúde; 2008.

BRASIL. Ministério da Saúde. Secretaria de Atenção à Saúde. Departamento de Atenção Básica. Política Nacional de Atenção Básica. Departamento de Atenção Básica. Brasília (DF): Ministério da Saúde; 2012.

BRASIL. Ministério da Saúde. Secretaria de Atenção à Saúde. Departamento de Atenção Básica. Política Nacional de Saúde Bucal. Departamento de Atenção Básica. Brasília (DF): Ministério da Saúde; 2004.

CONSTAND, M.K. et al. Scoping review of patientcentered care approaches in healthcare. BMC Health Services Research. v.14, p.271, 2014.

GOETZ, K. et al. Effectiveness of a quality management program in dental care practices. BMC Oral Health. v.14, p.41, 2014.

GONÇALVES, V.B. Percepções de Profissionais e Usuários com Relação à Odontologia na Estratégia de Saúde da Família. Dissertação de Mestrado em Odontologia em Saúde Coletiva. Faculdade de Odontologia de Piracicaba da Unicamp. Piracicaba, 2011.

KONTOPANTELIS, E.; ROLAND, M.; REEVES, D. Patient experience of access to primary care: identification of predictors in a national patient survey. BMC Family Practice. v.11, n.61, 2010.

MOHAMMED, S. et al. Performance evaluation of a health insurance in Nigeria using optimal resource use: health care providers perspectives. BMC Health Services Research. v.14, p.127, 2014.

MOIMAZ, S.A.S., et al. Satisfaction and perception of SUS's users about public health care. Physis, v.20, n.4, p.1419-1440, 2010.

MOIMAZ, S.A.S., et al. Oral health assessment protocol in primary care. RGO, v.63, n.4, p.446-454, 2015.

MOIMAZ, S.A.S., et al. Avaliação do usuário sobre o atendimento odontológico no Sistema Único de Saúde: uma abordagem à luz da humanização. Cienc Saude Colet. Artigo no prelo. Disponível em: http://www. cienciaesaudecoletiva.com.br/artigos/artigo_int.php?id_ artigo $=15307$. Acesso em: 20 jul 2016. 
NORA, C.R.D.; JUNGES, J.R. Política de humanização na atenção básica: revisão sistemática. Rev Saúde Pública. v.47, n.6, p.1186-200, 2013.

PAIM, J.S. Uma análise sobre o processo da Reforma Sanitária brasileira. Saúde em Debate, v. 33, n. 81, p. 2737, 2009.

PEDROSA, I.C.F.; CORRÊA, A.C.P.; MANDÚ, E.N.T. Influências da Infraestrutura de Centros de Saúde nas Práticas Profissionais: Percepções de Enfermeiros. Cienc Cuid Saude. v.10, n.1, p.58-65, 2011.

RICCI, N.A. et al. O hospital-escola de São Carlos: análise do funcionamento por meio da satisfação dos usuários. Cienc Saude Colet. v.16, n. Suppl 1, p. 1125-1134, 2011.

SERAPIONI, M.; SILVA, M.G.C. Avaliação da qualidade do Programa Saúde da Família em municípios do Ceará. Uma abordagem multidimensional. Cienc Saude Colet., v.16, n.11, p.4315-4326, 2011.

SOARES, S.C.M. Avaliação Da Saúde Bucal Na Atenção Básica: A Perspectiva Do Usuário. Dissertação mestre em Odontologia, Odontologia Preventiva e Social. UFRN. 2007.

TAVARES, R.P., et al. A organização do acesso aos serviços de saúde bucal na estratégia de saúde da família de um município da Bahia. Saúde em Debate. v. 37, n. 99, p. 628635, 2013.

VAN STRALEN, J.C; et al. Percepção dos usuários e profissionais de saúde sobre atenção básica: comparação entre unidades com e sem saúde da família na Região Centro-Oeste do Brasil. Cad. Saúde Pública, v.24, n.Sup1, p.148-158, 2008. 